\title{
Effect of pregnancy in myopia progression: the SUN cohort
}

\begin{abstract}
Purpose Previous studies have suggested that pregnancy may induce myopia progression. However, no longitudinal study with a large sample size and long-term follow-up has assessed this association. Our objective was to investigate whether pregnancy was related to mid- or long-term myopic refraction changes.

Patients and methods A prospective study was conducted in a Mediterranean cohort. The study included 10401 women (20-50 years old) from the SUN Project. SUN project is a multipurpose, prospective, and dynamic cohort of university graduates conducted in Spain. The recruitment of participants started in 1999 and it is permanently open. All participants in this cohort had university studies. Participants were followed up for a period of up to 14 years, and pregnancy and refractive changes were assessed through baseline and biennial questionnaires. Pregnancies and myopia were repeatedly assessed in each biennial follow-up questionnaire during a total of 14 years of follow-up.

Results Pregnancy was inversely associated with the risk of myopia development or progression during each of the 2 years periods, with fully adjusted hazard ratio $=0.61 ;(95 \%$ confidence interval $=0.49-$ 0.75 ) after adjusting for known potential confounders.

Conclusion To our knowledge this is the first large-longitudinal assessment in young adult women, showing that pregnancy is inversely associated with myopia development or progression. Further studies are needed to confirm these epidemiological findings.

Eye (2017) 31, 1085-1092; doi:10.1038/eye.2017.24; published online 17 March 2017
\end{abstract}

\section{Introduction}

Myopia is the most common type of refractive error world-wide. ${ }^{1,2}$ The balance of the axial
A Fernández-Montero',2, M Bes-Rastrollo ${ }^{1,2,3}$, J Moreno-Montañés ${ }^{2,4}$, L Moreno-Galarraga ${ }^{2,5}$ and MÁ Martínez-González 1,2,3,6

length of the eye, the optical power of the cornea and the lens determine the refractive status., ${ }^{2,3}$ Myopia is the most common refractive error among young adults ${ }^{4}$ and its prevalence has significantly increased, producing important social and economic consequences. ${ }^{2}$ The prevalence of myopia in adults varies across populations and ethnicities. A meta-analysis conducted by the Eye Diseases Prevalence Research Group estimated the crude prevalence rates for myopia (-1.0 dioptre (D) or less) as 25.4\% (CI 95\%: 24.46-26.39), in the United States, with similar prevalence in Western Europe and slightly lower in Australia. ${ }^{5}$ Therefore, myopia represents a large burden of ophthalmological disease, generates a considerable economic impact, and it is currently an important public health problem. ${ }^{2,6-8}$

The specific risk factors for myopia are still unclear, but evidence suggest a multifactorial cause with interplay between genetic and environmental factors. ${ }^{1,2,9}$ Inherited factors, specially important for syndromic high myopias, may include a personal susceptibility to the environmental circumstances and to classical risk factors such as age, race, near work, computer use, and educational level. ${ }^{1,10-14}$

In women, pregnancy has not been clearly identified as a myopia risk factor, but there is a classical belief that pregnancy is accompanied by physiological changes that temporally increase the refractive error. ${ }^{15-17}$ In fact, currently it is considered advisable to postpone any changes in eyeglass prescriptions until several weeks postpartum. ${ }^{18}$ Corrective procedures such as laser refractive surgery are also contraindicated during pregnancy, and current recommendations are that women should avoid pregnancy 1 year after surgery. ${ }^{19,20}$

Since women fertility age range often overlaps in time with the increase of some of the risk factors and with the refractive surgery, it would be beneficial to investigate whether pregnancy is an independent risk factor for the debut or progression of myopia.

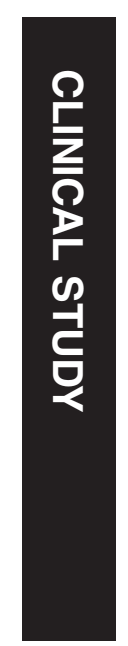

${ }^{1}$ Department of Preventive Medicine and Public Health, University of Navarra, Pamplona, Spain

${ }^{2}$ IdiSNA-Health Research Institute of Navarra, Pamplona, Spain

${ }^{3}$ CIBERobn, Instituto de Salud Carlos III, Madrid, Spain

${ }^{4}$ Department of Ophthalmology, Clínica Universidad de Navarra, Pamplona, Spain

${ }^{5}$ Department of Pediatrics, Complejo Hospital de Navarra, Pamplona, Spain

${ }^{6}$ Department of Nutrition, Harvard TH Chan School of Public Health, Boston, USA

\section{Correspondence:}

A Fernández-Montero, Department of Preventive Medicine and Public Health, Medical School-Clínica Universidad de Navarra, University of Navarra, C/Irunlarrea 1, 31008 Pamplona, Navarra, Spain Tel: +34 948 425600; Fax: +34 948425649. E-mail: afmontero@una.es

Received: 22 December 2015

Accepted in revised form: 31 January 2017 Published online: 17 March 2017 
Nevertheless, very few large studies with a prospective design have been published. A review of the literature regarding ocular changes associated with pregnancy revealed little mention of refractive changes. ${ }^{21-23}$ Only small series of cases have analysed refractive shifts during pregnancy, with contradictory results. ${ }^{16-18}$ To the best of our knowledge, this is the first longitudinal study with a large sample size assessing the association between pregnancy and myopia onset or progression.

\section{Materials and methods}

\section{Study population}

The SUN project is a multipurpose, prospective, and dynamic cohort of young adult university graduates conducted in Spain. The SUN Project study methods have been previously published in detail. ${ }^{24}$ The recruitment of participants started in 1999 and is permanently open. Mailed questionnaires are used to gather baseline characteristics and information on diet, lifestyles and new medical diagnoses of disease every 2 years. All participants included in the SUN cohort have university studies and over $50 \%$ are health professionals.

After the exclusion of men and recently recruited participants (we included only women with a minimum of 2-year follow up), and including only women between 20 to 50 years old (mean age at baseline: 32.9 SD: 8.1 ) we had follow-up information on 12143 women. We defined retention rate as the ratio of women who completed at least one follow-up questionnaire, among all the women who completed the initial questionnaire. Our retention rate was $86 \%$. We excluded 1742 women who provided no information on pregnancy or myopia. Thus, the effective sample size for our analyses was 10401 women. Each woman was followed up for a minimum of 2 years and a maximum of 14 years, since the fist participants were included in 1999. Each woman answered a follow- up questionnaire biennially, therefore there were seven possible periods of biennial follow up. However, we were able to analyse repeated measurements of both exposure and outcome for six of the seven periods due to the unavailability of information on pregnancies during the first period. Women who became pregnant more than once during the total study period, were included repeatedly in the analyses, using appropriate statistical procedures of repeated measurements (time-dependent Cox models) to account for autocorrelation.

Figure 1 shows how the total study period (14 years) was sub-divided in 7 biennial periods of follow up, between each questionnaire. The first follow-up period, between baseline questionnaire (Q0) and second questionnaire (Q2), referred as 'follow up period 0-2 years', is the only period where information about pregnancies was not collected, therefore, no measurements for this period were included in the timedependent Cox models. As shown in Figure 1, each biennial period was analysed using repeated measurements. Pregnancy and myopia information were assessed in each follow-up questionnaire, and delivery date was always confirmed to be previous to the reported date of myopia onset or progression within each of the six follow-up periods (Figure 1). The cases of myopia referred before the delivery date in each specific follow-up period were not considered as an outcome.

\section{Assessment of pregnancy (exposure)}

Pregnancies were assessed in each biennial follow-up questionnaire. All questionnaires, with the exception of Q2, included the following question: Have you been diagnosed by a doctor of a pregnancy since the last questionnaire? If so, please report estimated due data (month/year). This is the reason why we had only six, instead of seven follow-up periods.

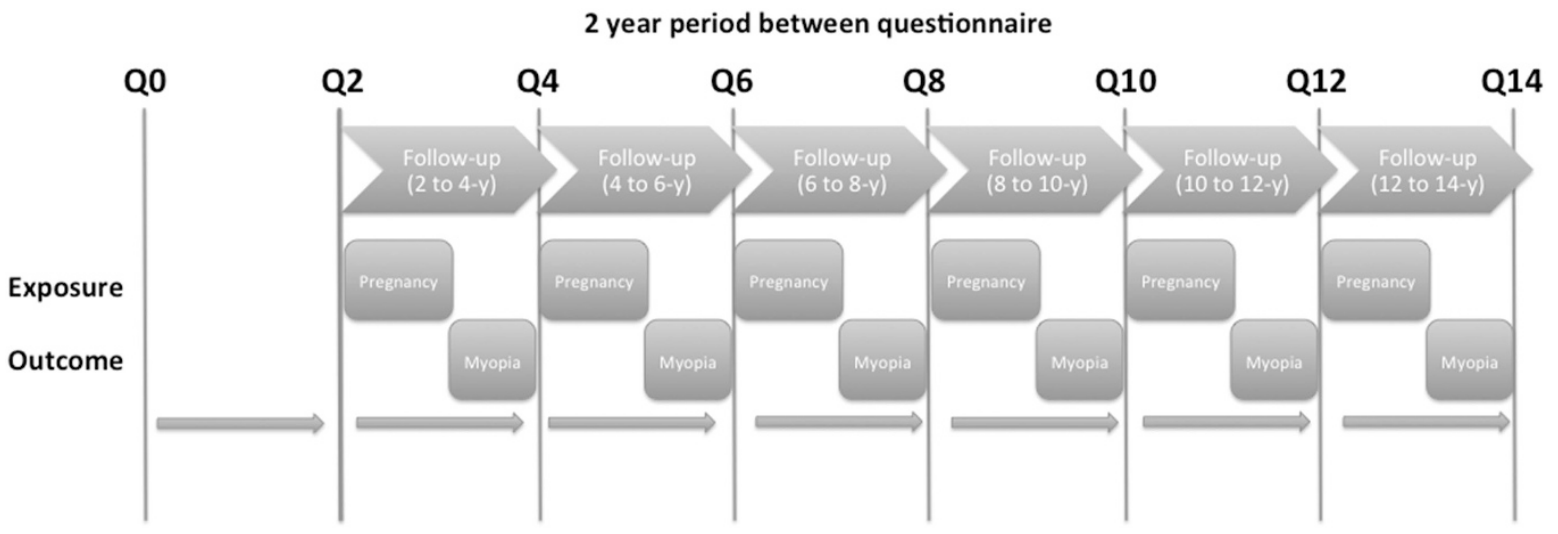

Figure 1 Follow-up periods in the SUN cohort. 


\section{Assessment of myopia (outcome)}

All follow-up questionnaires included the following question: Have you been diagnosed by a medical doctor of new-onset myopia or a progression of 0.5 or more dioptres in myopia, since the last questionnaire you filled in?

Participants who responded affirmatively to this question were considered as incident cases. Participants were considered a case of myopia progression when they reported a new diagnosis of myopia or an increase in myopia of at least - 0.50D in one eye, as assessed by a board certified ophthalmologist or university certified optometrist.

\section{Assessment of other variables}

The baseline questionnaire collected information on sociodemographic variables including marital status, anthropometric characteristics, lifestyle and health related habits, including adherence to Mediterranean Diet, smoking habits, total energy intake, alcohol intake, sleeping behaviour, time of television watching, computer use, outdoors physical activity, and medical history.

Time spent on outdoor physical activities was estimated by the information obtained from the questionnaires. Time (in hours/week) spent in different outdoors activities such as walking, gardening, running, cycling, or playing outdoors sports was obtained in the baseline questionnaire. To estimate the total time of outdoors physical activity per week, we summed the time spent in all this outdoors activities. Unfortunately, it was not possible to determine changes in these activities during pregnancies or maternity leaves, as we had only recorded the baseline information.

\section{Statistical analysis}

For each participant within each of the six follow-up periods we computed person-years of follow up, from the date of returning the baseline questionnaire to the date of myopia onset or progression, death, or to the date of returning the last follow-up questionnaire, whichever came first.

Time-dependent Cox regression models (proportional hazards models) were fitted to assess the relationship between the previous pregnancy in that follow-up period and myopia onset or progression. Therefore, the effect of pregnancy on myopia onset or progression was studied every two years. Total Study length was for over 14 years, and the mean follow up time within each of the six follow-up periods was of 1.63 years after a pregnancy (or after the previous questionnaire for women who were not pregnant in that period).
We estimated hazard ratios (HR) and their 95\% confidence intervals (CI) across two categories (pregnancy or no-pregnancy) using a time-dependent Cox regression analysis with age as the underlying time variable. Subsequently, we fitted a multivariable model additionally adjusting for age, BMI, total energy intake, Mediterranean Diet (Trichopoulou's score), smoking habits, computer use, educational level, sleeping behaviour, time of television watching, and physical activity. We also present a graphical display ot the data, showing the Kaplan-Meier plot, using the inverse probability weight method to account for potential confounding factors.

Six separated Cox regression model were assessed after stratifying by each of the six follow-up periods, adjusting for the same variables as mentioned above for the fully adjusted multivariable model. We conducted a sensitivity analysis, excluding all women who had a previous pregnancy before the baseline questionnaire (Q0). We also conducted an additional analysis were we stratified the data by age ( $\leq 30$ vs $>30$ years old).

We tested for proportionality of hazards by testing of non-zero slope in a generalized linear regression of the scaled Schoenfeld residuals on time (Grambsch and Therneau (1994)). We found a $P$-value $=0.22$ using the command 'stphtest, rank detail' implemented in STATA 12.0. With the final sample size analysed, we calculated an estimated power for Cox regression of 0.84 ensuring adequate power. All $P$-values are two-tailed and statistical significance was set at $P<0.05$. We used STATA 12.0 for all analyses. The Human Research Ethical Committee at the University of Navarra approved the study protocol. Voluntary completion of the first questionnaire was considered as informed consent. Our Institutional Review Board specifically approved this consent process.

\section{Results}

From the 10401 women included in the analyses, 3180 reported a pregnancy during the study period.

Table 1 shows the distribution of baseline characteristics of the participants according to pregnancy. Women who underwent a pregnancy were more likely to be younger, active smokers, drank less alcohol, slept less hours, and on average did less physical activity than non-pregnant women. They were also slightly more exposed to computer use, and watched more TV. Educational level was high in both groups as the SUN project includes only university graduates. Also, two Supplementary Tables are provided, showing the baseline characteristics of women excluded from our analysis due to missing data (Supplementary Table annex 1) and the baseline characteristics of participants according to a previous pregnancy at the time of inclusion (Supplementary Table annex 2). 
Table 1 Baseline characteristics of participants according to pregnancy

\begin{tabular}{|c|c|c|}
\hline & No pregnancy & Pregnancy \\
\hline$N$ & 7221 & 3180 \\
\hline \multicolumn{3}{|l|}{ Person-years in follow-up period } \\
\hline $2-4$ years & 13638 & 2523 \\
\hline 4-6 years & 23347 & 4744 \\
\hline $6-8$ years & 27983 & 6038 \\
\hline $8-10$ years & 32067 & 6698 \\
\hline 10-12 years & 30939 & 5181 \\
\hline $12-12$ years & 13143 & 1059 \\
\hline Age $^{\mathrm{a}}$ & $35.3(8.5)$ & $28.4(4.2)$ \\
\hline $\mathrm{BMI}^{\mathrm{a}}$ & $22.2(3.1)$ & $21.4(2.6)$ \\
\hline Exposure to computer (h/week) ${ }^{a}$ & $13.6(14.7)$ & $14.7(15.8)$ \\
\hline TV watching $(\mathrm{h} / \text { week })^{\mathrm{a}}$ & $11.3(9.1)$ & $12.2(9.7)$ \\
\hline \multicolumn{3}{|l|}{ Smoking status, n (\%) } \\
\hline Current & $1649(22.8)$ & $800(25.2)$ \\
\hline Former & $1865(25.8)$ & $586(18.4)$ \\
\hline Total energy intake (Kcal./day) $)^{\mathrm{a}}$ & $2505.3(892.1)$ & $2545.7(991.3)$ \\
\hline Outdoors physical activity (h/week) ${ }^{\mathrm{a}}$ & $5.2(5.4)$ & $5.2(5.8)$ \\
\hline Sleeping $<7 \mathrm{~h} /$ day, $n(\%)$ & $996(13.8)$ & $364(11.5)$ \\
\hline Insomnia, $n(\%)$ & $920(12.7)$ & $456(14.3)$ \\
\hline Alcohol intake $(\mathrm{g} / \mathrm{d})^{\mathrm{a}}$ & $4.1(6.1)$ & $3.8(5.2)$ \\
\hline Mediterranean dietary score $(0-8)^{a}$ & $3.6(1.5)$ & $3.5(1.4)$ \\
\hline \multicolumn{3}{|l|}{ Educational level, n (\%) } \\
\hline Doctorate & $528(7.3)$ & $137(4.3)$ \\
\hline Master & $528(7.3)$ & $244(7.6)$ \\
\hline Postgraduate & $3227(45.3)$ & $1525(48.0)$ \\
\hline College & $2173(30.1)$ & $982(30.9)$ \\
\hline Others & $720(10.0)$ & $292(9.2)$ \\
\hline
\end{tabular}

${ }^{\mathrm{a} S D}$.

The association between pregnancy and the risk of myopia onset or progression is shown in Table 2. Pregnancy was inversely associated with the risk of myopia development or progression, with fully-adjusted HR: 0.58 (95\% CI: 0.49-0.69) after adjusting for known potential confounders. Also an adjusted HR was estimated after a multivariable analysis stratifying by age and the inverse association remained statistically significant, suggesting that the inverse association between pregnancy on myopia was not related to age: HR: 0.59 (95\% CI: 0.47-0.76) in women under 30 years of age, and HR: 0.64 (95\% CI: 0.43-0.97) in women older than 30 years. This inverse association was more evident in younger women, but the $P$-value of the product-term for statistical interaction was not significant $(P=0.68)$.

Figure 2 shows a graphical display of the data, using a Kaplan-Meier plot, where the incidence of myopia onset or progression among pregnant and non-pregnant women progressively diverged.

Table 3 shows the association between pregnancy and risk or myopia onset or progression in each period of follow up. In all the periods included, there was an
Table 2 HR and $(95 \% \mathrm{CI})$ of myopia progression $(-0.5 \mathrm{D})$ according to a previous pregnancy. Cox models for repeated measurements

\begin{tabular}{lccc}
\hline & $\begin{array}{c}\text { No } \\
\text { pregnancy in } \\
\text { any } \\
\text { follow-up } \\
\text { questionnaire }\end{array}$ & $\begin{array}{c}\text { At least one } \\
\text { pregnancy in any } \\
\text { follow-up } \\
\text { questionnaire }\end{array}$ & $\mathrm{P}$ \\
& & & \\
\hline Incident cases of myopia & 1159 & 116 & \\
Person-years & 21524 & 3656 & \\
Crude HR & 1 (ref.) & $0.60(0.51-0.72)$ & $<0.001$ \\
Age-adjusted HR & 1 (ref.) & $0.57(0.48-0.68)$ & $<0.001$ \\
Multivariable-adjusted & 1 (ref.) & $0.58(0.49-0.69)$ & $<0.001$
\end{tabular}

$\mathrm{HR}^{\mathrm{a}}$

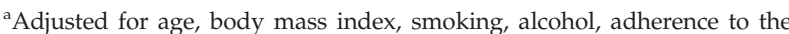
Mediterranean diet, outdoors physical activity, time of television watching, exposure to computer, educational level, sleeping hours, and total energy intake.

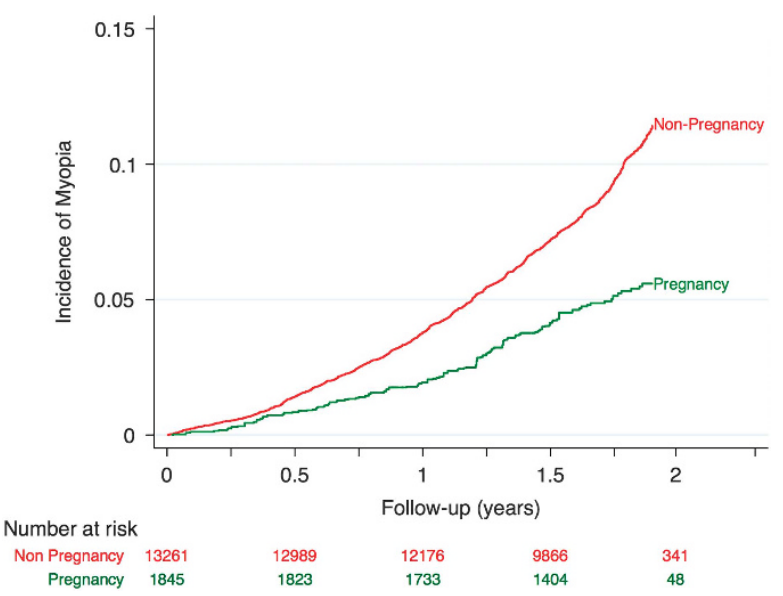

Figure 2 Kaplan-Meier estimates of the Incidence of myopia in the SUN cohort.

inverse association, but when we stratified by period of follow-up, it was only statistically significant for the three first periods of follow-up.

A likely explanation of the inverse association between pregnancy and myopia progression is the higher exposure to outdoor activities in pregnant women during their maternal leaves. Time of outdoors physical activity of 6.9 vs $5.2 \mathrm{~h}$ per week $(P=0.002)$ in pregnant woman compared to non-pregnant woman.

A sensitivity analysis was also assessed, excluding the women who had a previous pregnancy before the baseline questionnaire (Q0). After excluding these 3.979 women, 6422 women were included and with the multivariable analysis the risk of myopia onset or progression after a second pregnancy even decreased. HR: 0.55 (95\% CI: 0.44-0.70).

Finally, Figure 3 shows the HRs of myopia onset or progression, according to the number of pregnancies 
Table 3 HR and $(95 \% \mathrm{CI})$ of myopia on set or progression at the different follow-up periods

\begin{tabular}{lccc}
\hline & & & \\
& No pregnancy in the follow-up questionnaire & At least one pregnancy in the follow-up questionnaire \\
\hline Follow-up (2-4 years) $^{\mathrm{a}}$ & 1 (ref.) & $0.54(0.40-0.73)$ & $\mathrm{P}$ \\
Follow-up (4-6 years) $^{\mathrm{a}}$ & 1 (ref.) & $0.56(0.37-0.84)$ & $<0.001$ \\
Follow-up (6-8 years) $^{\mathrm{a}}$ & 1 (ref.) & $0.43(0.24-0.76)$ & 0.005 \\
Follow-up (8-10 years) $^{\mathrm{a}}$ & 1 (ref.) & $0.79(0.48-1.30)$ & 0.004 \\
Follow-up (10-12 years) $^{\mathrm{a}}$ & 1 (ref.) & $0.73(0.36-1.52)$ & 0.349 \\
Follow-up (12-14 years) $^{\mathrm{a}}$ & 1 (ref.) & $0.50(0.06-3.85)$ & 0.404 \\
\hline
\end{tabular}

adjusted for age, body mass index, smoking, alcohol, adherence to the Mediterranean diet, Outdoors physical activity, time of television watching, exposure to computer, educational level, sleeping hours, and total energy intake.

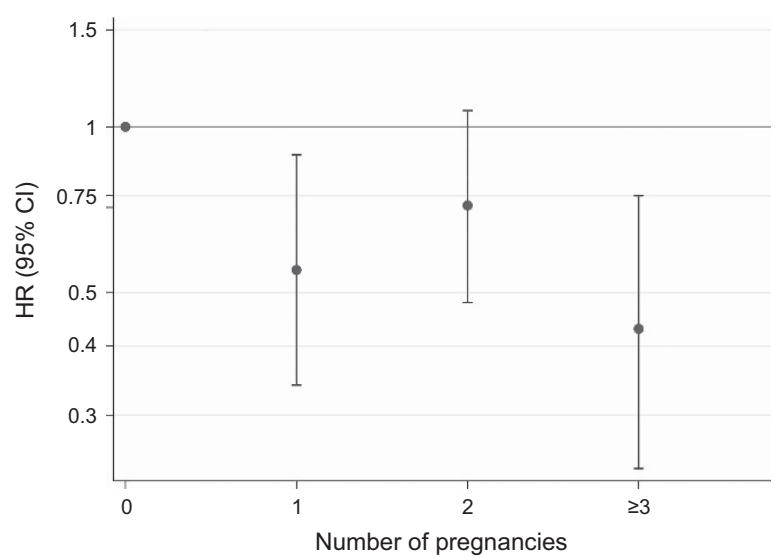

Figure $3 \mathrm{HR}$ and $(95 \% \mathrm{CI})$ of myopia onset or progression according to the number of pregnancies referred during total follow-up period.

referred during the total follow up period. It is shown how pregnancies have a protective effect on myopia onset or progression. A lower risk of myopia onset or progression was found in women with one pregnancy (HR $=0.55$; 95\% CI: 0.34-0.89) HR of 0.72 (95\% CI: $0.48-$ 1.07 ) was found in women with two pregnancies, and the lower risk was for those who referred three or more pregnancies (HR: 0.43 ; 95\% CI: $0.24-0.75$ ). Sensitivity analyses were also conducted under several assumptions, including multiple imputations of data for missing values, finding a very similar HR of 0.58 (95\% CI: 0.49-0.69).

\section{Discussion}

In our young adults female population of university graduates we did not find any evidence to support that pregnancy might be associated with an increased risk of myopia onset or progression. In fact, our findings showed exactly the opposite and supported a fairly strong inverse association, suggesting that pregnant women may have a lower risk of developing myopia onset or progression than their non-pregnant counterparts.
Age is an important and well-known factor in myopia progression, that's why we have adjusted and stratified our results by age. Moreover, we always used age as the underlying time variable in our Cox regression models. We used 30 years as a cutoff for stratified analyses, because it was previously estimated that this age was the age where myopia risk has a change in tendency (the risk decreases from 20 to 30 and then it slightly increases). Our results did not change with this analysis, showing that age does not act as an effect modifier of the inverse relation between pregnancy and myopia.

When the association between the cumulative number of pregnancies and myopia was studied, the highest inverse association with myopia (HR: 0.43 95\% CI: 0240.75 ) was observed for women with the highest number of pregnancies.

We also did a sensitivity analysis, excluding women who had a previous pregnancy before the baseline questionnaire $(\mathrm{Q} 0)$. The reason for this sensitivity analysis was the assumption that participants, who had been pregnant before baseline data collection, may have developed myopia or undergone changes in their myopia, prior to the baseline data being recorded.

Notwithstanding, the results of this sensitivity analysis were essentially the same.

We also adjusted our analysis according to hours/week of outdoor physical activity. It has been recently studied and published in recent literature the relationship between outdoors activities and a significant reduction in myopic progression. ${ }^{25,26}$

There is less information and inadequate knowledge regarding refractive errors related to pregnancy. Due to the high prevalence of myopia and its important socioeconomic consequences, the influence of pregnancy on myopia progression demands special attention.

During pregnancy, changes occur in the cardiovascular, hormonal, metabolic, hematologic, and immunologic system..$^{27}$ Undoubtedly, this adaptation process has an impact on the ocular system. Some authors have suggested that water retention during pregnancy induces changes on corneal thickness and curvature, and these changes could modify the corneal refractive index. ${ }^{28}$ 
Hormonal changes may also play a role since progestins and estrogens increase the permeability of the crystalline lens to water, thus reducing the refractive index. ${ }^{15}$

In any case, the observation that pregnant women have unstable refractions is a reality. Pizzarello et al ${ }^{16}$ reported in a case-control study with 12 pregnant women, that $25 \%$ of them noticed reduced night vision but all reported spontaneous resolution after delivery. On the other hand, small case series have reported no changes in refraction during pregnancy. Park et al ${ }^{17}$ followed up prospectively 24 women during their pregnancies and found an increase in corneal curvature during the second and third trimesters, but no significant permanent refractive change. Akar et al ${ }^{29}$ also evaluated 88 pregnant women and did not report significant differences. Manges et $a l^{16}$ followed 38 non-pregnant and 93 pregnant women and found that refractive errors did not change significantly during pregnancy.

This background, of contradictory results, has also invaded the field of refractive surgery mainly affecting corneal procedures (photorefractive keratectomy (PRK), and laser in situ keratomileusis). ${ }^{30,31}$ There is an appeal for women with a refractive error to consider refractive surgery nowadays. Classical recommendations for women considering refractive surgery include avoiding pregnancy for one year after surgery, and to have a stable documented refraction postpartum. ${ }^{15}$ The rationale for this recommendation is that first of all, the cornea after PRK may be more sensitive to hormonal changes during pregnancy leading to alterations on corneal wound healing, which could induce sub-epithelial haze, ${ }^{31}$ and that, it is generally believed, there is an increased risk of myopia progression during pregnancy. Therefore young fertile women seeking a possible refractive surgery need better and more solid information about the adequate timing of this surgery, according to their future pregnancies. We do not know the specifically physiological reason to explain our results: it can be due to water retention or other hormonal changes during pregnancies. But the increased time outdoors spent by women during their maternal leave and the fewer hours that they spent at work are very likely explanations of the observe inverse association. In any case, it is probably a multi-causal process. New epidemiological studies specifically focused on this issue and taking into account all these variables, should be conducted in order to better clarify these results.

Our findings showed that pregnancy was associated with a reduced risk of myopia onset or progression $(\mathrm{HR}=0.58 ; 95 \% \mathrm{CI}=0.49-0.69)$. The magnitude of this inverse association persisted after several degrees of control for potential confounders and several sensitivity analyses (Table 2).
All previous studies assessing this relation between pregnancy and myopia, involved $<100$ participants observed for short periods of follow-up. Probably, the refractory changes described were physiologic changes related to pregnancy that could later on have reverted to normality. This is why the time point when refractive changes are measured could be critical to the results found, and this could be a likely explanation for the apparently contradictory results reported.

Our study has several limitations to be considered when interpreting our findings. A methodological limitation is the potential recall error associated with selfreported data for onset or increase of myopia. Also, as the design of our cohort recruits only highly educated participants, and mostly all Caucasian, it could induce a selection bias, and further studies should be done in a more random sample of subjects, to be able to generalize our findings. However, this is a highly educated and cooperative cohort and we have evidence of the high quality and validity of the self-reported data provided. In fact several validations study have been done to confirm the high quality of the self-reported data of our participants. ${ }^{32-40}$ Another potential weakness of our study is the lack of information on myopia severity at baseline and we did not adjust or estimated for baseline myopia as we did not collect this information at baseline (Q0), consequently there is a possibility for uncontrolled confounding related to pre-existed myopia that we cannot account for. The literature provides limited data on the risk of myopia progression for different amounts of baseline myopia, but baseline myopia and its degree of severity are classically among the strongest determinants for myopia progression. ${ }^{41}$ Also, residual confounding could exist as we did not adjust for all the potential confounding variables such as parental history of myopia, or types or near work, or profession; however, our results did not change (they even became more robust) after adjusting for several confounding. It is possible that residual confounding by lower time of near work during the final months of pregnancy and during maternal leaves, and more time of external activities, might account for this inverse association that we found, and it could be a plausible explanation to these results, so these possible confounding factors should be taken into account in future studies. Also the definition of myopia or changes in myopia was based on criteria of wearing glasses, but not on measured refractive error.

Another possible limitation of our study is that conceivably a woman with a baby would be less likely to visit an eye doctor than a non-pregnant women and this could have induced selective detection bias. We analysed how many women had visited an ophthalmologist, and $27 \%$ of the pregnant women $v$ s $33 \%$ of the nonpregnant women underwent a check-up with an 
ophthalmologist $(P<0.001)$. But we also conducted a sub-study to conduct a small and simple bias analysis, and when we analysed only women reporting an ophthalmologist check-up there was still a significant reduction in the risk of myopia onset or progression in pregnant women compared to non-pregnant women (HR: 0.57; 95\%CI: 0.40-0.83).

Despite these limitations, our study is the first prospective cohort study, to our knowledge, with a large sample size and a long follow-up period that has investigated this association.

Our findings, if confirmed by further studies, can be of great relevance today. The most common age of females who seek refractive surgery is between 20 and 30 years, and this age coincides with the period of their lives that they are most likely to get pregnant.

Our study, conducted in a large cohort of highly educated Caucasians women, showed that pregnancy, was inversely associated with new myopia diagnosis or progression. Therefore, our results do not confirm the widespread idea that pregnancy may induce myopia progression. To confirm our findings, further longitudinal studies including a thorough assessment of baseline myopia are needed.

\section{Summary}

What was known before
Previous studies have suggested that pregnancy may
induce myopia progression. However, no longitudinal
study with a large sample size and long-term follow-up
has assessed this association.
What this study adds
To our knowledge this is the first large-longitudinal
assessment in middle-aged women, showing that
pregnancy is inversely associated with myopia
development or progression. Further studies are needed to
confirm these epidemiological findings.

\section{Conflict of interest}

The authors declare no conflict of interest.

\section{Author contributions}

AF contributed to data research, extracted data, performed data analysis and drafted the manuscript. MB performed data analysis and drafted the manuscript. JM reviewed data analysis, contributed to discussion and revision of the manuscript, and intellectual revision of the manuscript. LM reviewed data analysis, contributed to discussion and revision of the manuscript. MAM contributed to the design, the generation of the database, data analysis and intellectual revision of the manuscript.
All of them made substantial contributions to conception and design, acquisition of data, and approved the final version to be published.

\section{Acknowledgements}

We thank the other members of the SUN Group: Alonso A, Balaguer A, Barrio López MT, Basterra-Gortari FJ, Benito Corchón S, Beunza JJ, Carlos Chillerón S, Carmona L, Cervantes S, de Irala Estévez J, de la Fuente Arrillaga C, de la Rosa PA, Delgado Rodríguez M, Donat Vargas CL, Galbete Ciáurriz C, García López M, Gea Sánchez A, Goñi Ochandorena E, Guillén Grima F, Hernández A,

Lahortiga F, Llorca J, López del Burgo C, Marí Sanchís A, Martí del Moral A, Martín Calvo N, Martínez JA, NúñezCórdoba JM, Pimenta AM, Ruiz-Canela M, Ruiz Zambrana A, Sánchez Adán D, Sánchez-Villegas A, Sayón-Orea C, Toledo E, Toledo J, Vázquez Ruiz Z, and Zazpe I. The SUN Project has received funding from the Spanish Government-Instituto de Salud Carlos III, and the European Regional Development Fund (FEDER) (RD 06/0045, CIBER-OBN, Grants PI10/02658, PI10/02293, PI13/00615, PI14/01668, PI14/01798, PI14/01764, and G03/140), the Navarra Regional Government (45/2011, 122/2014), and the University of Navarra.

\section{References}

1 Pan C-W, Ramamurthy D, Saw S-M. Worldwide prevalence and risk factors for myopia. Ophthalmic Physiol Opt 2012; 32: 3-16.

2 Morgan IG, Ohno-Matsui K, Saw SM. Myopia. Lancet 2012; 379: 1739-1748.

3 Saw SM, Katz J, Schein OD, Chew SJ, Chan TK. Epidemiology of myopia. Epidemiol Rev 1996; 18: 175-187.

4 Konstantopoulos A, Yadegarfar G, Elgohary M. Near work, education, family history, and myopia in Greek conscripts. Eye (Lond) 2008; 22: 542-546.

5 Kempen JH, Mitchell P, Lee KE, Tielsch JM, Broman AT, Taylor HR et al. The prevalence of refractive errors among adults in the United States, Western Europe, and Australia. Arch Ophthalmol 2004; 122: 495-505.

6 Saw S-M, Gazzard G, Shih-Yen EC, Chua WH. Myopia and associated pathological complications. Ophthalmic Physiol Opt 2005; 25: 381-391.

7 Vu HT, Keeffe JE, McCarty CA, Taylor HR. Impact of unilateral and bilateral vision loss on quality of life. $\mathrm{Br} \mathrm{J}$ Ophthalmol 2005; 89: 360-363.

8 Grosvenor T. Why is there an epidemic of myopia? Clin Exp Optom 2003; 86: 273-275.

9 Mutti DO. Hereditary and environmental contributions to emmetropization and myopia. Optom Vis Sci 2010; 87: 406-413.

10 Fernández-Montero A, Olmo-Jimenez JM, Olmo N, Bes-Rastrollo M, Moreno-Galarraga L, Moreno-Montañés J et al. The impact of computer use in myopia progression: a cohort study in Spain. Prev Med 2015; 71: 67-71.

11 Hyman L, Gwiazda J, Hussein M, Norton TT, Wang Y, Marsh-tootle W et al. Relationship of age, sex, and ethnicity 
with myopia progression and axial elongation in the Correction of Myopia Evaluation Trial. Arch Ophthalmol 2005; 123: 977-987.

12 Teasdale TW, Fuchs J, Goldschmidt E. Degree of myopia in relation to intelligence and educational level. Lancet 1988; 2: 1351-1354.

13 Goss DA. Nearwork and myopia. Lancet 2000; 356: 1456-1457.

14 Mandel Y, Grotto I, El-Yaniv R, Belkin M, Israeli E, Polat U et al. Season of birth, natural light, and myopia. Ophthalmology 2008; 115: 686-692.

15 Omoti AE, Waziri-Erameh JM, Okeigbemen VW. A review of the changes in the ophthalmic and visual system in pregnancy. Afr J Reprod Health 2008; 12: 185-196.

16 Pizzarello LD. Refractive changes in pregnancy. Graefes Arch Clin Exp Ophthalmol 2003; 241: 484-488.

17 Schultz KL, Birnbaum AD, Goldstein DA. Ocular disease in pregnancy. Curr Opin Ophthalmol 2005; 16: 308-314.

18 Mehdizadehkashi K, Chaichian S, Mehdizadehkashi A, Jafarzadepour E, Tamannaie Z, Moazzami B et al. Visual acuity changes during pregnancy and postpartum: a crosssectional study in Iran. J Pregnancy 2014; 2014: 675792.

19 Talley AR, Assil KK, Schanzlin DJ. Patient selection and evaluation. In: Talamo JH, Krueqer RR (eds). The Excimer Manual: A Clinician's Guide to Excimer Laser Surgery. Little, Brown \& CoBoston: NY, USA, 1997 pp 35-51.

20 Serderavic O. Brightbill FSLaser in situ Keratomileusis: Results and Complications. In: Brightbill FS (ed) Corneal Surgery Theory, Technique \& Tissue, 3rd edn. Mosby: St Louis, MO, USA, 1999 p 809.

21 Weinreb RN, Lu A, Key T. Maternal ocular adaptations during pregnancy. Obstet Gynecol Surv 1987; 42: 471-483.

22 Dinn RB, Harris A, Marcus PS. Ocular changes in Pregnancy. Obstet Gynecol Surv 2003; 58: 137-144.

23 Barbazetto IA, Pizzarello LD. Ocular changes during pregnancy. Compr Ophthalmol Update 2007; 8: 155-167.

24 Segui-Gómez M, de la Fuente C, Vázquez Z, de Irala J, Martínez-González MA. Cohort profile: the 'Seguimiento Universidad de Navarra' (SUN) Study. Int J Epidemiol 2006; 35: 1417-1422.

25 Wu PC, Tsai CL, Wu HL, Yang YH, Kuo HK. Outdoor activity during class recess reduces myopia onset and progression in school children. Ophthalmology 2013; 120: 1080-1085.

26 Sherwin JC, ReacherMH, Keogh RH, Khawaja AP, Mackey DA, Foster PJ. The association between time spent out- doors and myopia in children and adolescents: a systematic review and meta-analysis. Ophthalmology 2012; 119: 2141-2151.

27 Thornburg KL, Jacobson SL, Giraud DG, Morton MJ. Hemodynamic changes in pregnancy. Semin Perinatol 2000; 24: 11-14.

28 Fatt I, Harris MG. Refractive index of the cornea as a function of its thickness. Am J Optom Arch Am Acad Optom 1973; 50: 383-386.
29 Akar Y, Yucel I, Akar ME, Zorlu G, Ari ES. Effect of pregnancy on intraobserver and intertechnique agreement in intraocular pressure measurements. Ophthalmologica 2005; 219: $36-42$.

30 Hefetz L, Gershevich A, Haviv D, Krakowski D, Eshkoly M, Nemet P. Influence of pregnancy and labor on outcome of photorefractive keratectomy. J Refract Surg 1996; 12: 511-512.

31 Sharif K. Regression of myopia induced by pregnancy after photorefractive keratectomy. J Refract Surg 1997; 13(5 Suppl): $445-446$.

32 Sánchez-Villegas A, Martínez JA, De Irala J, MartínezGonzález MA. Determinants of the adherence to an 'a priori' defined Mediterranean dietary pattern. Eur J Nutr 2002; 41: 249-257.

33 Bes-Rastrollo M, Pérez Valdivieso JR, Sánchez-Villegas A, Alonso A, Martínez-González MA. Validación del peso e índice de masa corporal autodeclarados de los participantes de una cohorte de graduados universitarios. Rev Esp Obes 2005; 3: 183-189.

34 Martínez-González MA, López-Fontana C, Varo JJ, SánchezVillagas A, Martínez JA. Validation of the Spanish version of the physical activity questionnaire used in the Nurses' Health Study and Health Professionals' Follow-up Study. Public Health Nutr 2005; 8: 920-927.

35 Alonso A, Beunza JJ, Delgado-Rodríguez M, MartínezGonzález MA. Validation of self reported diagnosis of hypertension in a cohort of university graduates in Spain. BMC Public Health 2005; 5: 94.

36 Sanchez-Villegas A, Schlatter J, Ortuno F, Lahortiga F, Pla J, Benito $\mathrm{S}$ et al. Validity of a self-reported diagnosis of depression among participants in a cohort study using the Structured Clinical Interview for DSM-IV (SCID-I). BMC Psychiatry 2008; 8: 43.

37 Román-Viñas B, Ribas Barba L, Ngo J, Martínez-González MA, Wijnhoven TM, Serra-Majem L. Validity of dietary patterns to assess nutrient intake adequacy. Br J Nutr 2009; 101: S12-S12.

38 Pons-Villanueva J, Segui-Gomez M. Validation of selfreported motor-vehicle crash and related work leave in a multi-purpose prospective cohort. Int J Inj Contr Saf Promot 2010; 17: 223-230.

39 Barrio-Lopez MT, Bes-Rastrollo M, Beunza JJ, FernandezMontero A, Garcia-Lopez M, Martinez-Gonzalez MA. Validation of metabolic syndrome using medical records in the SUN cohort. BMC Public Health 2011; 11: 867.

40 Fernández-Montero A, Beunza JJ, Bes-Rastrollo M, Barrio MT, de la Fuente-Arrillaga C, Moreno Galarraga L et al. Validación de los componentes del síndrome metabólico autodeclarados en un estudio de cohortes. Gac Sanit 2011; 25: 303-307.

41 Gwiazda JE, Hyman L, Norton TT, Hussein ME, MarshTootle W, Manny R et al. Accommodation andrelated risk factors associated with myopia progression and their interaction with treatment in COMET children. Invest Ophthalmol Vis Sci 2004; 45: 2143-2151.

Supplementary Information accompanies this paper on Eye website (http://www.nature.com/eye) 